\title{
Assessment of Rehabilitation Infrastructure in Peru
}

\section{( 国 Article in press ? )}

- Fuhs, A.K. ${ }^{\mathrm{E} E m a i l}$ Author,

- LaGrone, L.N.a,

- Moscoso Porras, M.G.bc,

- Rodríguez Castro, M.J.d,

- Ecos Quispe, R.L.e,

- Mock, C.N. ${ }^{a}$

- View Correspondence (jump link)

- $\quad$ UUniversity of Washington, Seattle, WA

- ' School of Physical Therapy, Peruvian University of Applied Sciences, Lima, Peru

- $\quad$ Association for the Development of Student Research in Health Sciences, San Marcos Major National University, Lima, Peru

- 'Cayetano Heredia Peruvian University, Lima, Peru

- eNational Institute of Neurologic Sciences, Lima, Peru

Hide additional affiliations_

\begin{abstract}
Objective: To assess rehabilitation infrastructure in Peru in terms of the World Health Organization (WHO) health systems building blocks. Design: Anonymous quantitative survey; questions were based on the WHO's Guidelines for Essential Trauma Care and rehabilitation professionals' input. Setting: Large public hospitals and referral centers and an online survey platform. Participants: Convenience sample of hospital personnel working in rehabilitation and neurology $(\mathrm{N}=239)$, recruited through existing contacts and professional societies. Interventions: Not applicable. Main Outcome Measures: Outcome measures were for 4 WHO domains: health workforce, health service delivery, essential medical products and technologies, and health information systems. Results: Regarding the domain of health workforce, $47 \%$ of physical therapists, $50 \%$ of occupational therapists, and $22 \%$ of physiatrists never see inpatients. Few reported rehabilitative nurses (15\%) or prosthetist/orthotists (14\%) at their hospitals. Even at the largest hospitals, most reported $\leq 3$ occupational therapists (54\%) and speech-language pathologists $(70 \%)$. At hospitals without speech-language pathologists, physical therapists (49\%) or nobody (34\%) perform speech-language pathology roles. At hospitals without occupational therapists, physical therapists most commonly (59\%) perform occupational therapy tasks. Alternate prosthetist/orthotist task performers are occupational therapists (26\%), physical therapists (19\%), and physicians (16\%). Forty-four percent reported interdisciplinary collaboration. Regarding the domain of health services, the most frequent inpatient and outpatient rehabilitation barriers were referral delays (50\%) and distance/transportation (39\%), respectively. Regarding the domain of health information systems, $28 \%$ reported rehabilitation
\end{abstract}


service data collection. Regarding the domain of essential medical products and technologies, electrophysical agents (88\%), gyms (81\%), and electromyography $(76 \%)$ were most common; thickened liquids (19\%), swallow studies (24\%), and cognitive training tools (28\%) were least frequent. Conclusions: Rehabilitation emphasis is on outpatient services, and there are comparatively adequate numbers of physical therapists and physiatrists relative to rehabilitation personnel. Financial barriers seem low for accessing existing services. There appear to be shortages of inpatient rehabilitation, specialized services, and interdisciplinary collaboration. These may be addressed by redistributing personnel and investing in education and equipment for specialized services. Further examination of task sharing's role in Peru's rehabilitation services is necessary to evaluate its potential to address deficiencies. (c) 2017 American Congress of Rehabilitation Medicine.

Author keywords

- Health information systems

- Health personnel

- Health services

- Interdisciplinary communication

- Occupational therapy

- Peru

- Physical and rehabilitation medicine

- Physical therapists

- Rehabilitation

- Rehabilitation nursing

- Speech-language pathology

- World Health Organization

- ISSN: 00039993

- CODEN: APMHA

- Source Type: Journal

- Original language: English

- DOI: 10.1016/j.apmr.2017.10.020

- Document Type: Article in Press

- Publisher: W.B. Saunders 